\title{
The Tangled1 Gene Is Required for Spatial Control of Cytoskeletal Arrays Associated with Cell Division during Maize Leaf Development
}

\author{
Ann L. Clearya and Laurie G. Smith ${ }^{b, 1}$ \\ aplant Cell Biology Group, Research School of Biological Sciences, Australian National University, GPO Box 475, ACT 2601, \\ Canberra, Australia \\ ${ }^{b}$ Department of Biology, Coker Hall CB 3280, University of North Carolina at Chapel Hill, Chapel Hill, North Carolina 27599- \\ 3280
}

The cytoskeleton plays a major role in the spatial regulation of plant cell division and morphogenesis. Arrays of microtubules and actin filaments present in the cell cortex during prophase mark sites to which phragmoplasts and associated cell plates are guided during cytokinesis. During interphase, cortical microtubules are believed to influence the orientation of cell expansion by guiding the pattern in which cell wall material is laid down. Little is known about the mechanisms that regulate these cytoskeleton-dependent processes critical for plant development. Previous work showed that the Tangled1 ( $\operatorname{Tan} 1$ ) gene of maize is required for spatial regulation of cytokinesis during maize leaf development but not for leaf morphogenesis. Here, we examine the cytoskeletal arrays associated with cell division and morphogenesis during the development of $\tan 1$ and wild-type leaves. Our analysis leads to the conclusion that Tan 1 is required both for the positioning of cytoskeletal arrays that establish planes of cell division during prophase and for spatial guidance of expanding phragmoplasts toward preestablished cortical division sites during cytokinesis. Observations on the organization of interphase cortical microtubules suggest that regional influences may play a role in coordinating cell expansion patterns among groups of cells during leaf morphogenesis.

\section{INTRODUCTION}

The cellular architecture of plant tissues is defined by a network of cell walls, which determine the shape, size, and position of each cell. It is well known that the formation and initial positioning of cell walls, as well as their patterns of expansion during organ growth, are influenced by the cytoskeleton. Cell walls are initiated through the action of a phragmoplast composed of microtubules (MTs) and actin filaments, which guide the deposition of vesicles containing cell wall material to the growing cell plate (Gunning, 1982; Staehelin and Hepler, 1996). Although the cell plate itself is initiated at the end of mitosis, its final position is established much earlier; this position is marked during prophase by a cortical array of MTs, the preprophase band (PPB; PickettHeaps and Northcote, 1966). More recently, it has been recognized that in many prophase cells, an actin band colocalizes with the MT PPB (Palevitz, 1987; Traas et al., 1987; Cleary et al., 1992). Although an accurate indicator of the fu-

\footnotetext{
${ }^{1}$ To whom correspondence should be addressed at the Department of Biology 0116, 9500 Gilman Drive, University of California at San Diego, La J olla, CA 92093-0116. E-mail Ismith@biomail.ucsd.edu; fax 619-534-7108.
}

ture location of the new cell wall, the PPB itself is a transient structure. At the end of prophase, both actin and MT components of the PPB disappear from the cell cortex, but cortical actin is retained elsewhere, resulting in the formation of an actin-depleted zone (ADZ), which persists at the former PPB site throughout mitosis and cytokinesis (Cleary et al., 1992; Liu and Palevitz, 1992; Cleary, 1995; Baluska et al., 1997).

A variety of observations has established that during cytokinesis, the phragmoplast is actively guided to the former PPB site (Ota, 1961; Gunning and Wick, 1985; Palevitz, 1986; Cho and Wick, 1989). The molecular mechanisms responsible for phragmoplast guidance and the essential features of the established division site required for this process are not known. One attractive hypothesis is that the PPB may guide the local deposition or formation of a more lasting landmark of some kind that guides the phragmoplast and associated cell plate to this site during cytokinesis (Mineyuki and Gunning, 1990). It is also possible that the ADZ itself provides a spatial cue for guidance of the phragmoplast, or that the remaining cortical actin is important for maintaining the localization at the division site of something else that is necessary for phragmoplast guidance. 
Cytoskeletal filaments, particularly MTs, have also been implicated in the control of cell shape via their influence on the pattern of cell wall deposition. Although the organization of interphase cortical MTs is related in a predictable way to cell shape and the pattern of cell expansion, very little is known about how their arrangement is controlled (reviewed in Williamson, 1991; Cyr, 1994). Many experimental treatments can rapidly change the arrangement of interphase cortical MTs, including the application of hormones, electrical fields, and mechanical forces, but it is not known how these treatments act or which treatments have the most direct effects on MT organization. Indeed, because so many interrelated factors can influence the organization of interphase cortical MTs, understanding what controls their organization has proven to be a very complex problem.

One approach to tackling unsolved problems concerning the cytoskeletal basis of cell division and morphogenesis is isolating and analyzing mutants that are affected in these processes. In recent years, this approach has begun to yield new information regarding genes and molecules required for spatial regulation of cell division and morphogenesis during plant development (e.g., Shevell et al., 1994; Traas et al., 1995). Among these, the tangled 1 ( $\tan 1)$ mutation was shown recently to disrupt the spatial control of cytokinesis during maize leaf development (Smith et al., 1996). In wildtype leaves, cells are rectangular and divide either transversely or longitudinally with respect to the long axis of the mother cell, as illustrated in Figure 1A. In tan 1 leaves, cells divide transversely, but rarely do they divide longitudinally; instead, they divide in a variety of abnormal orientations not seen in wild-type leaves (Figure 1B).

Observations on the effects of this mutation on cell division have raised many questions regarding the cytoskeletal basis of the $\tan 1$ mutant phenotype. For example, are division planes established during prophase as marked by the formation of PPBs and ADZs? If so, do these arrays form in orientations that predict the abnormal planes of cell division

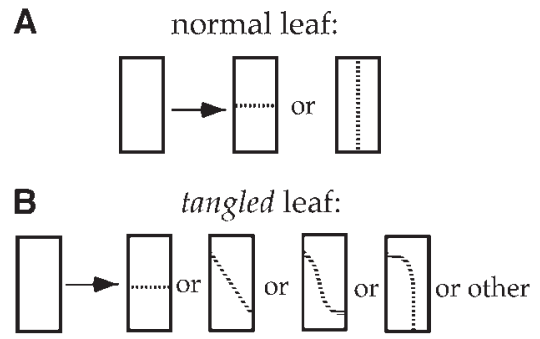

Figure 1. Cell Division Orientations in Wild-Type and tan1 Maize Leaf Epidermal Cells.

(A) Wild-type leaves. Cells divide either transversely or longitudinally with respect to the long axis of the mother cell.

(B) $\tan 1$ leaves. Cells divide transversely or in a variety of abnormal orientations, often laying down curved cell walls. found in developing mutant leaves, or are they oriented normally, implying that the division defects are due to events occurring at later stages of the cell cycle? Previous work with $\tan 1$ has also shown that despite the fact that cells in all tissue layers divide in abnormal orientations throughout the development of mutant leaves, these leaves acquire normal shapes (Smith et al., 1996). Can the organization of interphase cortical MTs in these normally shaped leaves composed of abnormally shaped cells give us insight into mechanisms of leaf morphogenesis?

To address these questions, we undertook an investigation of cytoskeletal organization in developing $\tan 1$ leaves compared with their wild-type siblings. The results of this analysis show that the Tan1 gene is required for the normal positioning of cytoskeletal arrays associated with cell division at all stages of the cell cycle but not for their formation. Analysis of interphase cortical MTs suggests that mutant leaves are of normal shape in part because of regional control of MT organization during interphase.

\section{RESULTS}

\section{Analysis of Cell Division Orientations in tan1-py1 Leaf Primordia}

Two $\tan 1$ mutant alleles are currently available: $\tan 1-M u 1$, which was isolated in 1990 from a stock containing active Mutator transposons and known to be caused by the insertion of Mul (L.G. Smith, unpublished results), and tan1-py1 (also called pyl or pigmy plant), a previously isolated mutation of spontaneous origin (Suttle, 1924). The phenotypes of plants homozygous for these two mutations appear very similar macroscopically, and the mutations have similar effects on the overall cell pattern of the leaf epidermis (data not shown), with the tan-pyl phenotype being generally more severe. Although tan1-Mu1 mutants were used for the previous analysis of cell division orientations during leaf development (Smith et al., 1996), we used tan1-py1 mutants for the cytoskeletal analysis because they express the phenotype more uniformly than do tan1-Mul mutants. Thus, to have a direct basis for comparison between the orientations of cytoskeletal arrays and those of new cell walls, it was necessary to examine cell division orientations in tan1-py1 mutants.

To do this, we used a whole-mount technique to visualize recently formed epidermal cell walls in leaf primordia that were $\sim 1 \mathrm{~cm}$ long. At this stage, wild-type leaves are $\sim 1 \%$ of their final length, most cells are rectangular, little cellular differentiation is apparent, as evidenced by the absence of guard mother cells and trichomes, and cells are dividing in both transverse and longitudinal orientations throughout the leaf (Sylvester et al., 1990; S mith et al., 1996). Wild-type and mutant primordia at this stage were stained with acriflavine under conditions in which this fluorescent dye stains both cell walls and nuclei, and epidermal anticlinal cell walls were 


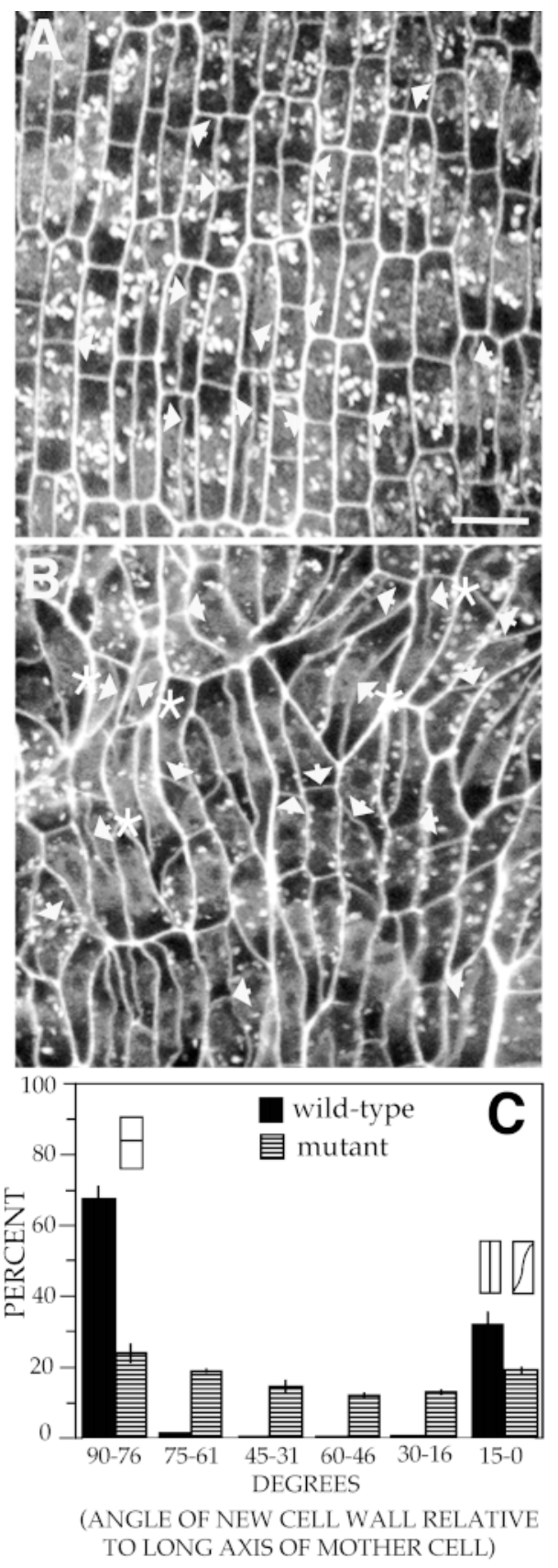

Figure 2. Orientations of Recently Formed Epidermal Cell Walls in Maize Leaf Primordia.

Low-intensity acriflavine staining was used to determine which cell walls had formed most recently (indicated by arrowheads). Brightly stained objects surrounding each nucleus are starch granules.

(A) Wild-type epidermis with new cell walls in transverse and longitudinal orientations.

(B) $\tan 1$ epidermis with new transverse and oblique cell walls that are either straight or curved. Arrowheads marked with asterisks indicate steeply oblique walls representing the majority of walls in the 0 to $15^{\circ}$ category shown in (C). examined by confocal laser scanning microscopy. Low-intensity cell wall staining was used to determine which walls had recently formed; examples are indicated by arrows in Figures $2 \mathrm{~A}$ and $2 \mathrm{~B}$. The orientation of each new wall was scored in relation to the long axis of the mother cell (Figure $2 \mathrm{C}$; see Methods for more details of this analysis).

In wild-type primordia, approximately two-thirds of new cell walls identified by this method are oriented transversely relative to the mother cell's long axis (at an angle of 76 to $90^{\circ}$ ), and the remaining one-third are oriented longitudinally $\left(0\right.$ to $15^{\circ}$ ) (Figures $2 \mathrm{~A}$ and $2 \mathrm{C}$ ). By comparison, previous analysis of new cell wall orientations in wild-type 1-cm leaf primordia by using scanning electron microscopy (SEM) showed approximately equal proportions of transverse and longitudinal divisions (Smith et al., 1996). This difference is presumably due to the different methodology used here to evaluate newly formed cell walls. Identification of new walls by SEM relies on assessment of wall shallowness. Longitudinal walls may remain shallow longer than transverse ones (perhaps because of different rates of longitudinal versus transverse cell expansion presumably required to modify wall depth), leading to an overestimate of the frequency of longitudinal cell divisions by this method. The acriflavine method uses fluorescence intensity as a direct measure of cellulose content, and because these results are in close agreement with those obtained for MT arrays presented below, we suggest that this is a more direct method for assessing new cell wall orientations.

In tan-py1 leaf primordia, a similar spectrum of new cell wall orientations can be observed by using the acriflavine staining method compared with that found previously in tanMul primordia by using SEM (Figures 1 and 2B). However, the frequency of transverse divisions observed in tan1-py1 primordia $(\sim 25 \%)$ is lower than that observed previously for $\tan 1-M u 1$ ( $\sim 50 \%$; Smith et al., 1996). This decrease in the proportion of cells dividing in a normal orientation and the corresponding increase in the proportion of cells dividing in abnormal orientations are in keeping with the more severe and more uniform phenotype of tan-py1 mutants, but the alternate methodology used here may also contribute to these differences, as discussed above. Although $\sim 20 \%$ of the walls observed in tan-pyl primordia form at an angle of 0 to $15^{\circ}$ relative to the long axis of the mother cell, very few of

(C) Quantitative analysis of new cell wall orientations relative to the long axes of parent cells. In wild-type cells, the majority of new walls are either transverse $\left(76\right.$ to $\left.90^{\circ}\right)$ or longitudinal $\left(0\right.$ to $\left.15^{\circ}\right)$. Mutant cells exhibit a wide range of new wall angles, with $\sim 25 \%$ being transverse $\left(76\right.$ to $\left.90^{\circ}\right)$ and the remainder having a wide variety of angles $\leqslant 75^{\circ}$ to the long axis of the parent cell. In mutant cells, the frequencies of new cell walls at all angles are significantly different from those in wild-type cells $(P<0.05)$. Error bars indicate standard errors. For the wild type, $n=1807$; for $\tan 1, n=1167$.

Bar in (A) $=20 \mu \mathrm{m}$ for (A) and (B). 
these are truly longitudinal. Due to the method used to assign the angles of new cell walls (see Methods), steeply oblique walls account for the majority of those in the 0 to $15^{\circ}$ category (see examples marked with asterisks in Figure $2 \mathrm{~B}$ ).

In summary, the results of the present analysis using acriflavine staining to examine the effects of tan-pyl on cell division orientations are broadly similar to those obtained in the previous analysis of tan-Mul by using SEM; however, they paint a somewhat different picture when the frequencies are considered. The previous analysis showed that the frequency of transverse divisions in tan-Mul primordia was almost unchanged compared with that of the wild type, whereas the frequency of longitudinal divisions was reduced from almost $50 \%$ in the wild type to $<5 \%$ in mutants. This result was interpreted to suggest that the Tan1 gene product may be selectively required for longitudinal cell divisions (Smith et al., 1996). Our analysis of tan1-pyl shows that this allele also produces a high frequency of abnormally oriented divisions but reduces the frequency of normally oriented divisions in both transverse and longitudinal classes. Thus, although this analysis confirms that the Tan1 gene product is required for cells to divide in normal orientations, the hypothesis that it is selectively required for longitudinal divisions is not supported.

\section{Analysis of MT Arrays Associated with Cell Division in Wild-Type and tan1 Leaf Primordia}

To determine how the $\tan 1$ mutation affects the formation and orientation of MT arrays associated with cell division, we needed an approach that would allow a large number of arrays to be examined in relation to the shape of the dividing cell. For this purpose, a whole-mount procedure was developed that permitted simultaneous visualization of fluorescently labeled MTs and DNA in epidermal cells of maize leaf primordia at the same 1-cm stage used for the new cell wall analysis (see Methods). This procedure permitted us to collect images, such as those shown in Figure 3A (wild type) and Figures 3B to 3D (mutant), showing a high frequency of dividing cells at various stages of the cell cycle. Altogether, $\sim 2200$ cells in prophase, mitosis, or cytokinesis were examined from a total of four mutant and four wild-type leaf primordia to determine the orientation of their MT arrays relative to the long axis of the dividing cell; the results of this quantitative analysis are shown in Figure 4.

As shown in Figure $4 \mathrm{~A}, 73 \%$ of PPBs in wild-type leaf primordia are transverse (oriented 76 to $90^{\circ}$ relative to the cell's long axis), whereas $22 \%$ are longitudinal (oriented 0 to $15^{\circ}$ relative to the long axis). These frequencies are in close agreement with those obtained for newly formed cell walls by using the acriflavine method (Figure $2 \mathrm{C}$ ). Only $2 \%$ of wild-type PPBs were found at oblique angles (16 to $75^{\circ}$ ), and only $2 \%$ were found in an asymmetrical position (outside of the middle third of the cell). Thus, the vast majority of PPBs in wild-type leaf primordia are symmetrically posi- tioned and are either transversely or longitudinally oriented. As illustrated in Figure 4B, 33\% of wild-type spindles are transversely oriented (i.e., the spindle axis is parallel to the long axis of the cell), whereas $10 \%$ are longitudinally oriented. Unexpectedly, the majority of spindles $(57 \%$ altogether) are obliquely oriented, indicating that the spindles rotate during mitosis in wild-type cells and that their orientations are not constrained by any prior definition of the division site, as indicated by the PPB. Analysis of phragmoplast orientations (Figure $4 \mathrm{C}$ ) indicates that phragmoplasts are repositioned during cytokinesis to "correct" for the oblique spindles: although oblique early phragmoplasts are often seen in wild-type cells (e.g., Figure 3A, arrow labeled with a circle), the vast majority of late phragmoplasts (those whose edges are within $2 \mu \mathrm{m}$ of the parental wall at both ends) are either transversely $(73 \%)$ or longitudinally $(24 \%)$ oriented. Thus, as would be expected, the distribution of late phragmoplast orientations (Figure $4 \mathrm{C}$ ) closely matches that of PPBs (Figure 4A) as well as that of new cell walls (Figure 2C).

In $\tan 1$ leaf primordia, cells with PPBs, spindles, and phragmoplasts were seen at approximately the same frequency as in wild-type primordia on a per cell basis as well as at similar relative frequencies. These arrays were seen at a variety of orientations relative to the long axis of the dividing cell, but they always appeared structurally normal (Figures 3B to 3D). Virtually all PPBs in mutant cells are straight, and $90 \%$ are positioned symmetrically (within the middle third of the cell). Thus, asymmetrically placed PPBs are found more frequently than they are in wild-type cells (10 versus $2 \%$ ) but are still a small minority. As might be expected from the very low frequency of longitudinal divisions within this population of cells, a 10 -fold decrease in the proportion of longitudinal PPBs was observed (2\%) when compared with the wild type $(22 \%)$. As illustrated in Figures $3 B$ to $3 \mathrm{D}$ and $4 \mathrm{~A}$, the vast majority of PPBs formed by mutant cells are transverse or slightly oblique $\left(80 \%\right.$ at a 76 to $90^{\circ}$ angle; $12 \%$ at a 61 to $75^{\circ}$ angle relative to the long axis). Notably, PPBs predicting the abnormally shaped and oriented new cell walls found in this population of cells were not observed.

The abnormal orientations of new cell walls seen in mutant cells appear to result mainly from events that occur during mitosis and cytokinesis. Spindle orientations in mutant cells are highly variable, indicating that spindle rotation occurs during mitosis in mutant cells as it does in wild-type cells (Figures $3 \mathrm{~B}$ and $4 \mathrm{~B}$ ). By the end of the cell cycle, only $28 \%$ of late phragmoplasts are transverse (Figure $4 C$ ). Thus, unlike the situation in wild-type cells, in which the orientations of late phragmoplasts closely match those of PPBs, a substantial proportion of mutant phragmoplasts fail to return to the transverse orientations occupied by most PPBs. As seen earlier in the analysis of new cell wall orientations (Figure $2 B$ ), many of the late phragmoplasts seen in mutant cells are curved (see arrows marked with asterisks in Figures $3 \mathrm{C}$ and $3 \mathrm{D}$ ). Indeed, the majority of late phragmoplasts in the 0 to $15^{\circ}$ class are curved and form at a steeply oblique angle 

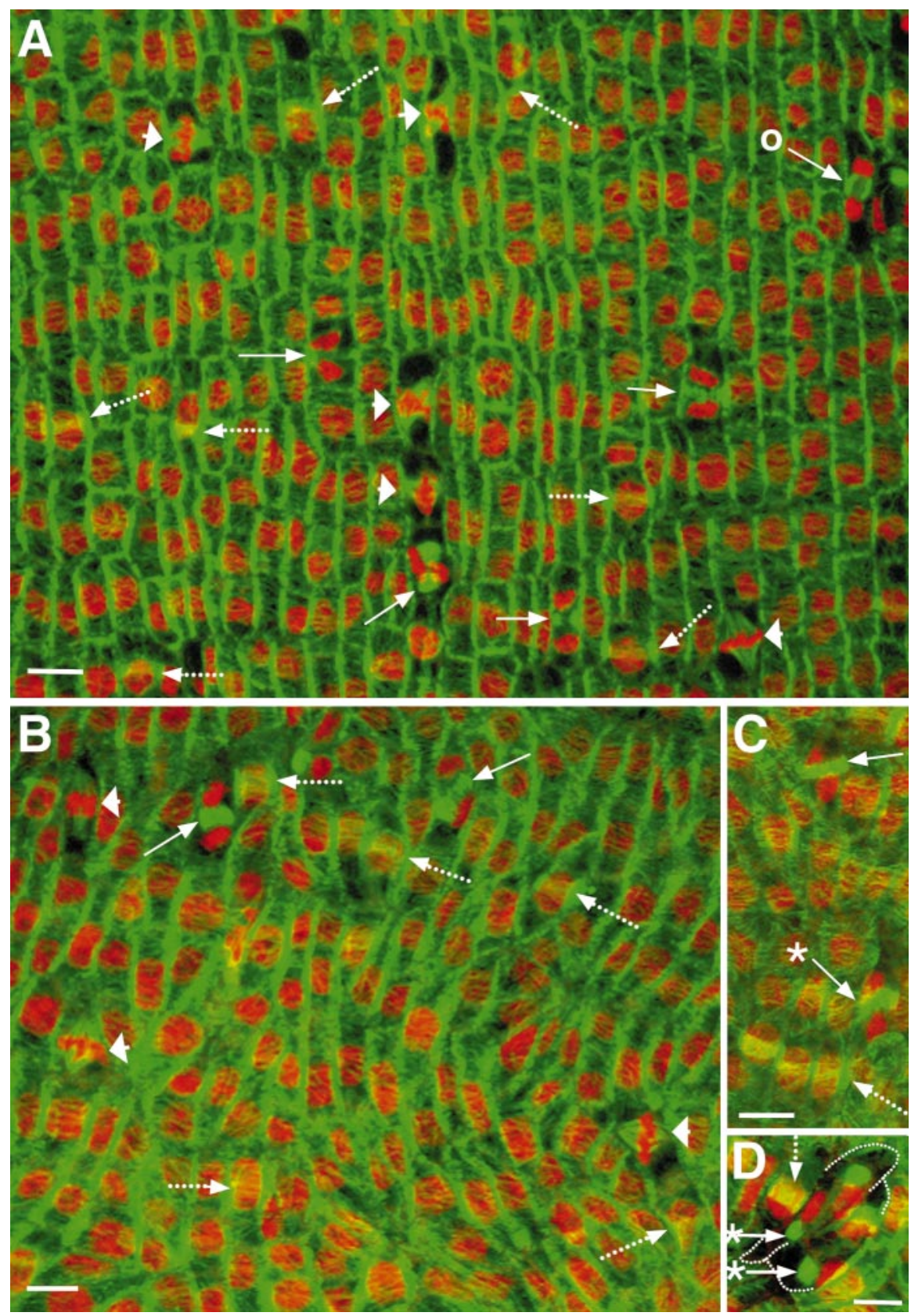

Figure 3. MTs and DNA in Epidermal Cells of Maize Leaf Primordia.

In (A) to (D), a surface section and midplane optical section of MTs are superimposed (shown in green) together with a midplane section through the nuclei/chromosomes (shown in red). PPBs are indicated by arrows with dotted stems, spindles with arrowheads, and phragmoplasts with solid-stemmed arrows. Bars in (A) to (D) $=10 \mu \mathrm{m}$.

(A) Wild type. PPBs are either transversely or longitudinally oriented, with the majority of spindles being obliquely oriented. Although oblique early phragmoplasts are seen frequently (arrow marked with a circle), late phragmoplasts are always transversely or longitudinally oriented.

(B) to (D) tan1 mutant. All PPBs shown are transverse to the cell's long axis, whereas spindle orientations are variable. Phragmoplasts without asterisks in (B) and (C) are straight and are either transversely or obliquely oriented. Phragmoplasts marked with asterisks are slightly curved in (C) or steeply oblique and curved in (D); dotted lines in (D) outline cell ends. 

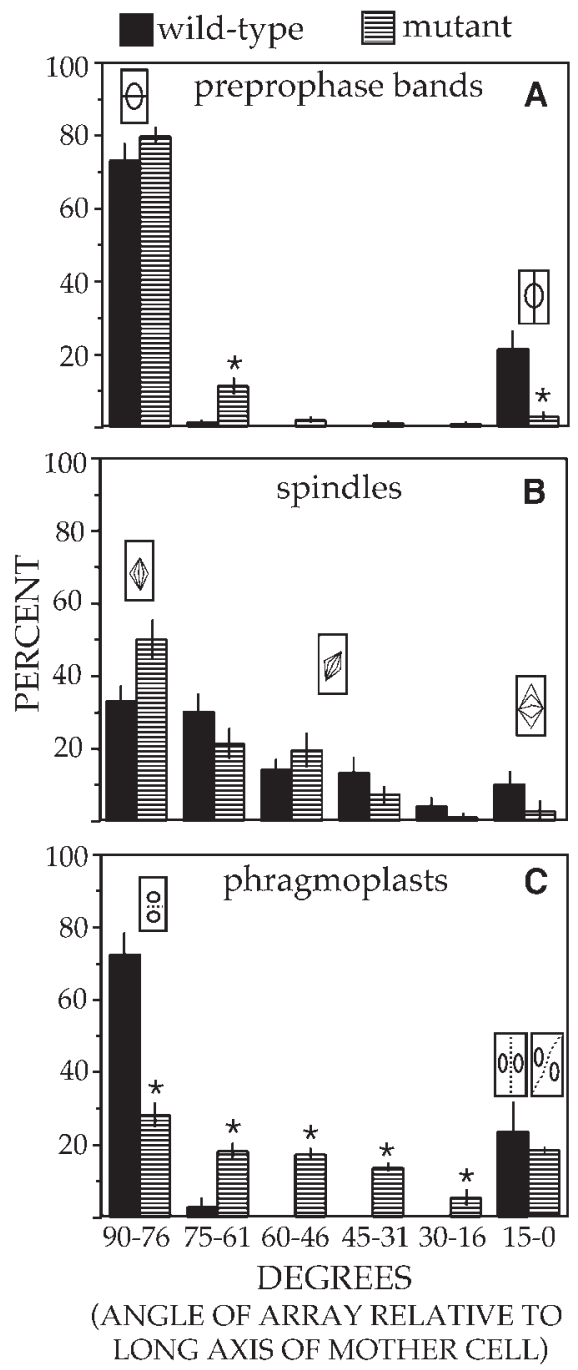

Figure 4. Quantitative Analysis of the Orientations of Mitotic MT Arrays in Epidermal Cells of Maize Leaf Primordia.

Error bars indicate standard errors. Asterisks indicate categories in which the results for mutant cells are significantly different from those for wild-type cells $(P<0.01)$.

(A) PPBs in wild-type cells are mainly transversely or longitudinally oriented; in $\tan 1$ cells, they are mainly transverse or slightly oblique in orientation; only $2 \%$ are longitudinal. For the wild type, $n=900$; for $\tan 1, \mathrm{n}=822$.

(B) Spindles are found at all angles in both wild-type and mutant cells. For the wild type, $n=115$; for $\tan 1, n=128$.

(C) Late phragmoplast orientations closely match those of PPBs in wild-type cells. In mutant cells, late phragmoplast orientations are highly variable and do not match those of PPBs. Mutant phragmoplasts in the 0 to $15^{\circ}$ class are mainly steeply oblique and curved. For the wild type, $n=134$; for $\tan 1, n=135$. relative to the cell's long axis. Thus, the distribution of late phragmoplast orientations and shapes seen in mutant cells (Figure 4C) corresponds closely to that shown in Figure 2C for new cell walls in this population of cells.

\section{Analysis of Actin Arrays Associated with Cell Division in Wild-Type and $\tan 1$ Leaf Primordia}

Although variability in the results from different studies makes it more difficult to generalize about rearrangements of the actin cytoskeleton associated with cell division compared with MTs, certain features of actin organization during cell division have been widely observed and, if present, provide useful markers for establishment of the division plane and maintenance of the division site during mitosis and cytokinesis. Our analysis of MT PPBs showed that they are structurally normal in tan1 mutant cells but do not form in the same orientations as they do in wild-type cells. We sought to extend these observations by asking whether mutant cells in prophase are also normal with respect to actin PPBs, and if so, whether actin and MT bands coincide. Furthermore, because our results showed a lack of correspondence in mutant cells between the orientations of MT PPBs and those of late phragmoplasts, we wanted to know whether ADZs form and are maintained throughout mitosis and cytokinesis, and if so, how their positions relate to those of phragmoplasts. To answer these questions, we examined the organization of the actin cytoskeleton throughout the cell cycle in mutant and wild-type leaf primordia. To permit direct comparisons between actin and MT organization, we used a double-labeling procedure that permitted simultaneous visualization of MTs and actin in individual cells (see Methods).

Examination of actin arrays in each of three wild-type and three mutant leaf primordia revealed no differences in actin organization at any stage of the cell cycle. Figure 5 illustrates cells at various stages of the cell cycle that are double labeled for MTs (Figures 5A, 5C, 5E, 5G, and 5I) and actin (Figures 5B, 5D, 5F, 5H, and $5 \mathrm{~J}$ ). Both mutant and wild-type cells have all classes of $\mathrm{F}$-actin typically found during interphase: randomly oriented cortical actin filaments, longitudinal subcortical actin bundles, and actin bundles found within transvacuolar cytoplasmic strands and associated with the periphery of the nucleus (Figure 5B). As cells approached mitosis (early preprophase), cortical actin filaments became more uniformly oriented in the transverse direction before the formation of actin bands (data not shown).

Examination of cortical actin in 120 preprophase-prophase cells from wild-type and mutant leaves combined revealed three distinct arrangements of actin. Previous work has established that as prophase proceeds, MT PPBs become progressively narrower (Wick, 1991b). Because the three prophase actin arrangements that we observed could be related to MT PPB widths, they appear to represent three 
sequential stages. Consistent with our results for MT PPBs (Figure 4A), these actin-MT arrangements were observed in both transverse and longitudinal orientations in wild-type cells but mostly in transverse orientations in mutant cells.

Actin filaments and MTs are coaligned in wide bands during the initial stage (stage I) of PPB formation (arrows marked $I$ in Figures $5 \mathrm{~A}$ and $5 \mathrm{~B}$ ). At this stage, the width of the actin PPB usually exceeds that of the MT PPB (19.0 \pm $9.5 \mu \mathrm{m}$ for MT PPBs versus $22.9 \pm 9.3 \mu \mathrm{m}$ for actin PPBs).
The high variability in band width during this stage reflects the wide range of values within which MT PPBs narrow while intact actin PPBs are retained. During stage II, MT PPBs are consistently narrower than at stage I (14.2 \pm 4.3 $\mu \mathrm{m})$, and the density of actin diminishes in the central area of the band (arrows marked II in Figures $5 \mathrm{~A}$ and $5 \mathrm{~B}$ ). This area, a putative early $A D Z$, is generally narrower than the corresponding MT PPB $(10.7 \pm 3.8 \mu \mathrm{m})$. Parallel actin filaments, which are remnants of the former band, flank the
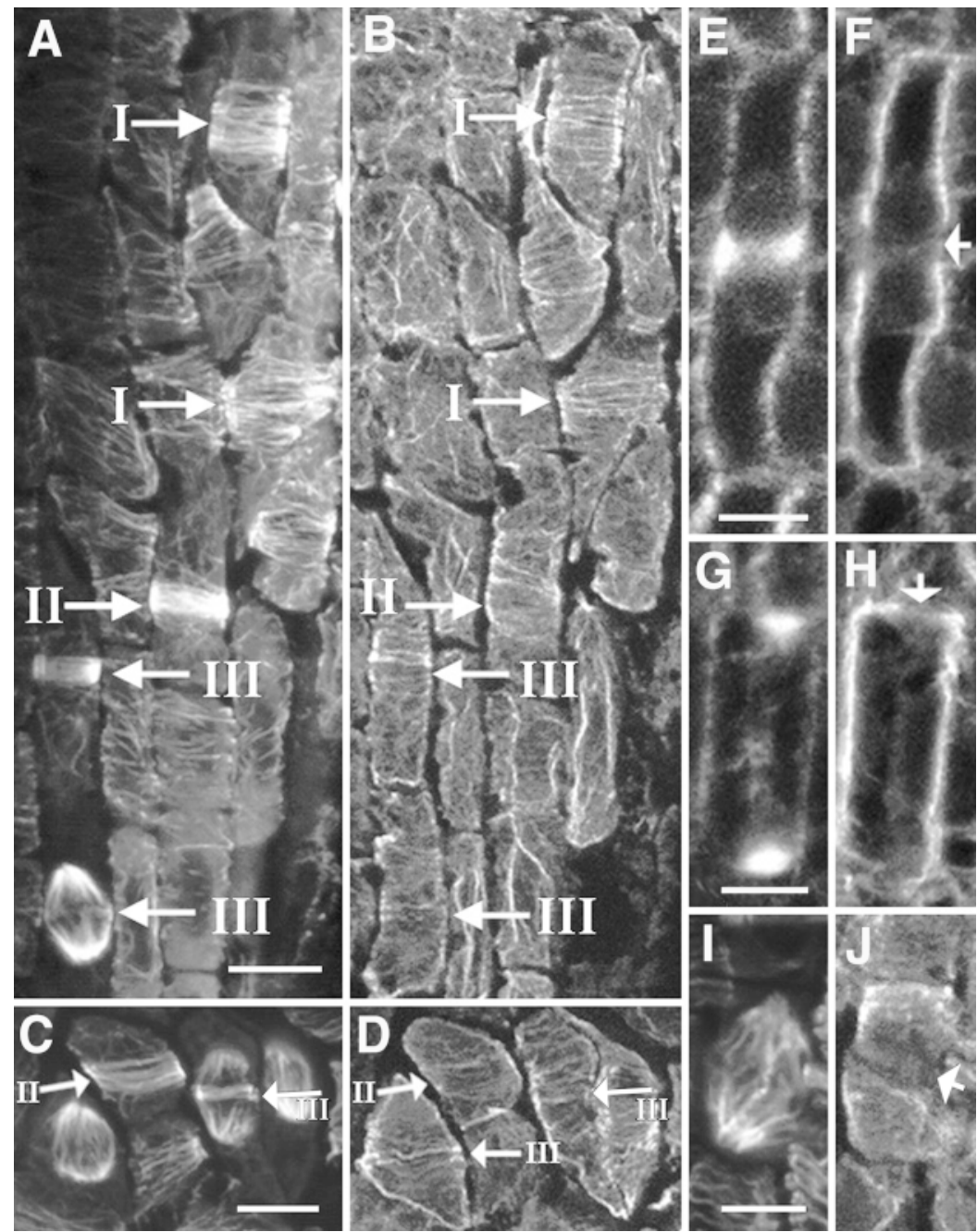

Figure 5. Double Labeling of MTs and Actin in Epidermal Cells of Maize Leaf Primordia.

MTs are shown in (A), (C), (E), (G), and (I), and actin is shown in (B), (D), (F), (H), and ( ) ). Bars in (A), (C), (E), (G), and (I) = $10 \mu \mathrm{m}$ for (A) to ( ) . (A) and (B) Surface view of tan1 cells displaying stages I to III of PPB development.

(C) and (D) Surface view of tan1 cells displaying stages II and III of PPB development; the leftmost cell in (C) and (D) shows a stage III actin arrangement that persists in the absence of a corresponding MT PPB.

(E) and (F) Wild-type cell in midplane view showing a transverse phragmoplast and an ADZ (arrow).

(G) and (H) Wild-type cell in midplane view showing a longitudinal phragmoplast and an ADZ (arrow).

(I) and $(\boldsymbol{U}) \tan 1$ cell in surface view showing an anaphase spindle and an ADZ (arrow). 
ADZ and extend past the edges of the MT PPB. In some cases, the width of the remnant band is equivalent to the stage I actin band; however, in others, the parallel filaments extend over larger areas of the cell cortex and are indistinguishable from non-PPB cortical actin filaments. During stage III of PPB development, both the MT PPB $(8.2 \pm 3.1$ $\mu \mathrm{m})$ and ADZ $(5.8 \pm 3.0 \mu \mathrm{m})$ are even narrower, with the ADZ again being consistently narrower than the MT PPB (arrows marked III in Figures $5 A$ to $5 D$ ). A pair of thick actin cables frequently delineate the edges of the ADZ from the persistent cortical F-actin (see Figure 5D), which in some cases is still arranged in parallel arrays; however, in other instances, it is faintly stained and randomly organized. Several clear examples were observed in which the stage III actin arrangement persisted after the disappearance of the MT PPB (e.g., leftmost cell in Figures 5C and 5D).

Although ADZs were clearly and consistently observed in both wild-type and $\tan 1$ mutant cells during prophase, they were not consistently observed during mitosis and cytokinesis in either wild-type or mutant cells. Those ADZs that were observed demonstrate that wild-type cells can maintain ADZs in both transverse (Figures $5 \mathrm{E}$ and $5 \mathrm{~F}$ ) and longitudinal (Figures $5 \mathrm{G}$ and $5 \mathrm{H}$ ) orientations and in the transverse orientation in the mutant (Figures $5 \mathrm{I}$ and $5 \mathrm{~J}$ ). However, the low frequency at which ADZs were identified during mitosis and cytokinesis meant that little information could be obtained concerning the maintenance or orientation of division sites at these stages of the cell cycle.

In summary, this analysis demonstrates that actin bands and ADZs are present during prophase in both tan 1 mutant and wild-type cells. Furthermore, in $\tan 1$ cells, they have the same temporal and spatial relationship to MT PPBs observed in wild-type cells, that is, they appear to undergo the same maturation process during prophase, and they always coincide with MT PPBs. Thus, we conclude that actin PPBS and ADZs are affected by the tan 1 mutation in the same way as the PPB of MTs: they are present and structurally normal but form mainly in transverse orientations instead of forming both transversely and longitudinally.

\section{Analysis of Interphase Cortical MT Arrays in Wild-Type and $\tan 1$ Leaf Primordia}

Previous analysis of the tan 1 mutant phenotype showed that despite the high frequency of abnormally oriented cell divisions occurring throughout leaf development in all tissue layers, the morphogenesis of mutant leaves proceeds normally: they grow more slowly, but their overall shapes from primordial stages through maturity are very similar to those of wild-type leaves of the same length (Smith et al., 1996). These observations raise the question of how leaves of normal shape are formed by a population of cells dividing in abnormal orientations and having abnormal shapes. Perhaps, regardless of the irregularities in their individual shapes, the expansion of mutant leaf cells can be oriented properly in relation to the organ or region in which they reside.

It is well established that interphase cortical microtubules generally are aligned perpendicular to the major axis of cell expansion (reviewed in Williamson, 1991; Cyr, 1994). During the growth of wild-type leaves, cells are rectangular and their long axes are aligned; therefore, if cortical MTs are oriented perpendicular to the long axis of each cell, they will be coaligned throughout broad areas of the growing leaf. In expanding $\tan 1$ leaves, however, cell axes are frequently skewed relative to one another. If the expansion of these cells is controlled at a regional level, we might expect interphase cortical MTs in mutant cells to be coaligned among groups of cells with divergent shapes rather than having an alignment consistently related to cell shape. To address this issue, we examined interphase cortical microtubule arrays in wild-type and mutant leaf primordia.

A representative image of cortical microtubules in a wildtype leaf primordium is shown in Figure $6 \mathrm{~A}$. Although there is variation in the degree and orientation of alignment observed, cortical MTs of most interphase cells are predominantly transversely aligned. Occasionally, cortical MTs are aligned predominantly parallel to the long axis of the cell (Figure 6A, arrow). It is likely that such cells either recently completed a longitudinal division or were about to divide longitudinally at the time of fixation, because they are most often seen in areas in which surrounding cells are at various stages of the longitudinal division process. In some cells, little or no alignment of interphase cortical MTs could be discerned.

Images of cortical MT arrays in two different areas of tan1 mutant leaf primordia are shown in Figures $6 \mathrm{~B}$ and $6 \mathrm{C}$, illustrating considerable variation in the orientations of long cell axes and in the arrangements of interphase cortical MTs. Alignment of cortical MTs was observed within most cells; for many, the orientation of alignment is perpendicular to the cell's long axis (e.g., Figure 6B, upper right quadrant). Interphase cortical MTs generally are not coaligned across cell fields of the size shown in Figures $6 \mathrm{~B}$ and $6 \mathrm{C}$. However, $\mathrm{a}$ striking degree of coalignment typically is observed across boundaries at which shifts in cell orientation occur. For example, cortical MTs in the cells indicated with arrowheads in Figures $6 B$ and $6 C$ are better aligned with the MTs of neighboring cells than they are with the indicated cell's own transverse axis. Not all cells follow this rule: MTs in some cells are neither coaligned with MTs of neighboring cells nor transverse to the cell's long axis. In view of the observed combination of transversely aligned, regionally coaligned, and sometimes unpredictably arranged interphase cortical MTs, it seems remarkable that when mutant cells enter prophase, PPBs are virtually always oriented transversely (Figure 4A). Notably, this occurs even in areas in which the interphase cortical MTs are all oriented differently (e.g., Figure $6 \mathrm{~B}$, cell marked with large arrow). These observations suggest that there may be some regional influence on interphase cortical MT orientations, which helps to guide normal 


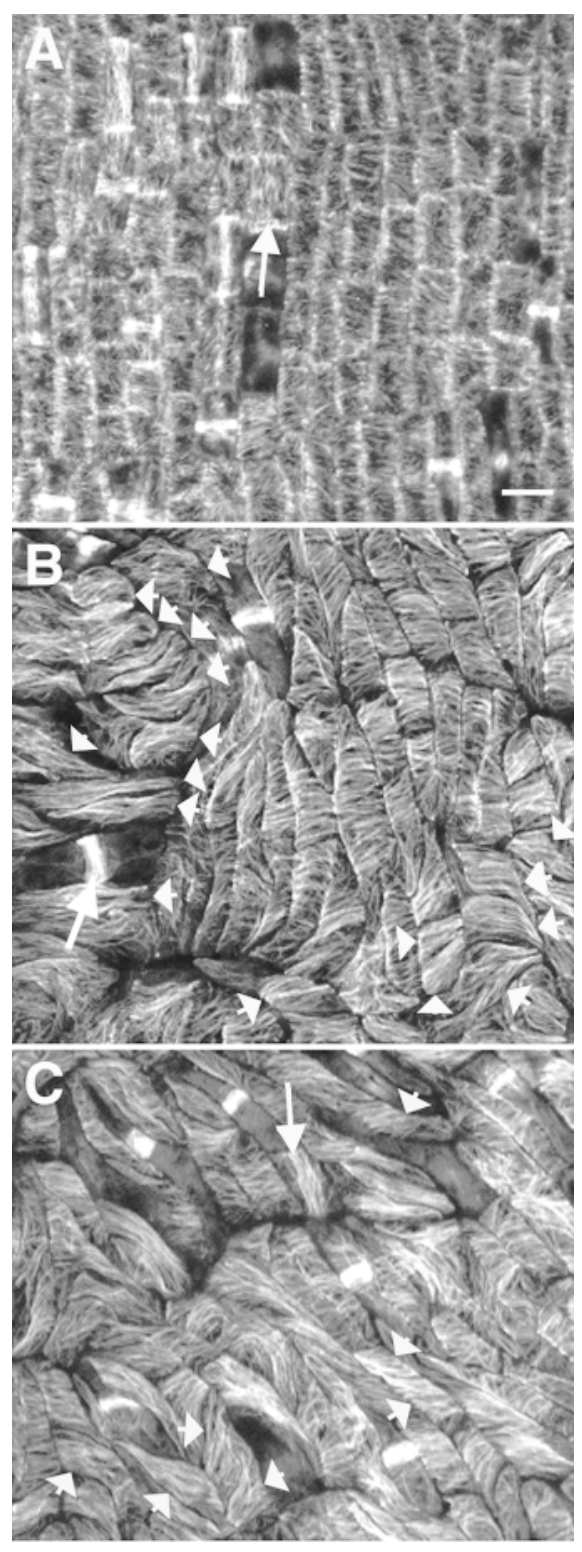

Figure 6. Cortical MTs in Epidermal Cells of Maize Leaf Primordia.

All images are single optical sections (surface views).

(A) Wild type. Most interphase cortical MTs are transverse to the cell's long axis. Occasionally, MTs are aligned parallel to the long axis of the cell (arrow).

(B) and (C) $\tan 1$ mutant. MTs are aligned within most cells. Arrowheads indicate cells whose MTs are better aligned with those of neighboring cells than they are with the cell's own transverse axis. The arrow in (B) indicates a PPB oriented transversely to the long axis of the cell, in contrast to the cortical MTs in the surrounding interphase cells. The arrow in (C) indicates a rare longitudinal PPB in a cell with square ends.

Bar in (A) $=10 \mu \mathrm{m}$ for (A) to (C). morphogenesis in mutant leaves but apparently has no impact on the choice of division plane in cells preparing to divide.

\section{DISCUSSION}

In a previous study, it was shown that $\tan 1$ causes cells to divide in abnormal orientations throughout maize leaf development (Figure 1; Smith et al., 1996). In this study, we analyzed how the $\tan 1$ mutation affects MT and actin arrays associated with the formation and positioning of new cell walls during leaf development. The data provide a better understanding of the role played by the Tan 1 gene in the spatial regulation of cytokinesis and also highlight newly recognized features of the spatial control of cytokinesis.

Analysis of cytoskeletal arrays associated with cell division in wild-type leaf primordia led us to infer the sequence of events depicted in Figure 7A. As expected from a large body of previous work showing that MT PPBS accurately predict planes of cell division in a wide variety of plant cells (reviewed in Wick, 1991a, 1991b), transverse and longitudinal MT PPBs were observed at similar relative frequencies to those of newly formed cell walls. Also consistent with previous studies (Kakimoto and Shibaoka, 1987; Palevitz, 1987; Traas et al., 1987; McCurdy and Gunning, 1990; Cleary et al., 1992; Liu and Palevitz, 1992; Cleary and Mathesius, 1996), we found that early in prophase, wide actin bands coincided with MT PPBs. Later in prophase, as the MT PPB narrowed, ADZs formed in positions that also coincided with the MT PPB. During mitosis, many spindles were found in transverse and longitudinal orientations, but the majority were oriented obliquely, indicating that rotation of the spindle during mitosis is a common occurrence in wild-type cells.

Although this high frequency of oblique spindles was unexpected, it was not surprising in view of earlier reports of spindle rotation followed by compensatory movements of the phragmoplast to position the cell plate at the site previously occupied by the PPB (e.g., Palevitz and Hepler, 1974; Palevitz, 1986). Similarly, in wild-type maize leaf primordia, spindle rotation during mitosis evidently is corrected later in the cell cycle, because late phragmoplasts and new cell walls are essentially always found in transverse or longitudinal orientations. In the few cases in which ADZs were clearly seen, they appeared to mark previously established division sites. We do not know why we were unable to detect ADZs consistently during later stages of the cell cycle, but it is likely that the method used was not ideal for this purpose. Studies demonstrating the persistence of ADZs throughout the cell cycle have most often utilized microinjection of rhodaminephalloidin (e.g., Cleary et al., 1992; Cleary, 1995); this method might reveal ADZs more consistently but would be technically difficult to implement in maize leaf primordia.

The corresponding sequence of events inferred from analysis of cytoskeletal arrays in $\tan 1$ mutant leaf primordia is 
A

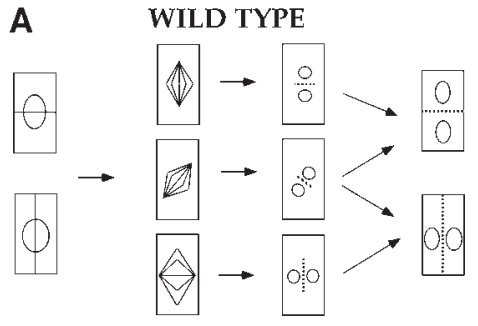

B

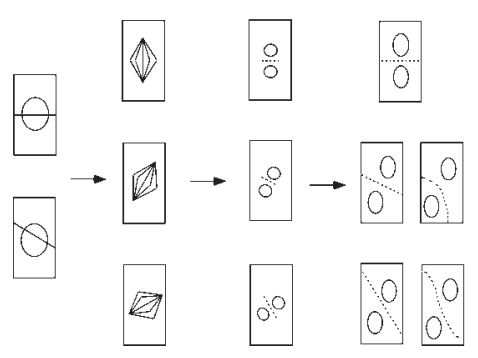

Figure 7. Schematic Illustration Showing the Sequence of Cytoskeletal Rearrangements Inferred for Wild-Type and $\tan 1$ Cells.

(A) Wild-type cells. PPBs are oriented either transversely or longitudinally. Some spindles are found in orientations similar to the PPBS, but most become obliquely oriented. The orientations of early phragmoplasts match those of the spindles. By the end of the cell cycle, the oblique angles of spindles/early phragmoplasts are corrected so that the final division planes are transverse or longitudinal.

(B) tan 1 mutant. PPBs are transverse or slightly oblique in orientation. Spindles and early phragmoplasts can remain transversely oriented, but the majority are obliquely oriented. By the end of the cell cycle, phragmoplast positions become highly variable, and the majority of new cell walls are not transverse.

shown in Figure 7B. During prophase, all cytoskeletal arrays observed in wild-type cells were also formed in mutant cells and appeared to be structurally normal: wide, early PPBs containing MTs and actin filaments, and late, narrow PPBs of MTs coinciding with ADZs. Thus, with respect to all prophase events that we were able to evaluate, division planes appeared to be established normally in mutant cells but formed mostly in transverse orientations. Therefore, the Tanl gene is apparently not required for division plane establishment per se but is required for the establishment of longitudinal division planes. One proposal to explain how cells choose a division plane is that they seek the shortest path that will halve the volume of the cell (Flanders et al., 1990; Lloyd, 1991). In the elongated cells of maize leaf primordia, this "default" choice would correspond to a transverse plane. Thus, establishment of any other division plane might require additional information, perhaps provided by or directly dependent on the Tan 1 gene product. However, it is also possible that the requirement for Tan1 to establish lon- gitudinal division planes is an indirect one. For example, establishment of longitudinal division planes may depend on some aspect of cell shape that is uncommon among mutant cells, such as a relatively low length/width ratio, or the presence of square cell ends. Indeed, the few longitudinal PPBs that we observed were always seen in cells that had square ends (e.g., Figure 6C, long-stemmed arrow).

As illustrated in Figure 7B, our results clearly show that most of the abnormally oriented divisions in mutant cells can be attributed to the frequent failure of phragmoplasts and associated cell plates to attach at cortical sites previously occupied by PPBs. During mitosis, approximately half of the spindles rotate to become oblique; by the end of cytokinesis, the majority of cells have non-transverse phragmoplasts in the variety of orientations and conformations illustrated, which are similar to those seen for new cell walls in mutant primordia. Thus, we conclude that Tan 1 is also required during cytokinesis for guidance of phragmoplasts and new cell walls to previously established division sites. Because nontransverse phragmoplasts outnumber oblique spindles, some of these phragmoplasts must arise in cells that had transverse spindles at mitosis, suggesting that aberrantly oriented divisions are not due exclusively to a failure to correct for spindle rotation. Indeed, using time-lapse video microscopy to watch living cells undergoing division, we observed a mutant cell in which the cell plate arose in a transverse orientation and drifted radically during cytokinesis to form an oblique, curved cell wall similar to the one illustrated in the lower right corner of Figure 7B (H.J. . Passas and L.G. Smith, unpublished observations). Our observations suggest that the requirement for $\operatorname{Tan} 1$ to achieve proper phragmoplast guidance applies not only to cells dividing longitudinally but to all dividing cells in the leaf primordium. It may be that many mutant cells divide transversely simply because this is a common outcome for an elongated cell that establishes a transverse division plane at prophase, even in the absence of phragmoplast guidance during cytokinesis. Because ADZs could not be identified consistently during mitosis and cytokinesis in either wild-type or mutant cells, we were unable to determine whether mispositioning of phragmoplasts during cytokinesis results from a failure to maintain the division site throughout the cell cycle or a failure of the phragmoplast to interact with it appropriately.

Our analysis of the cytoskeletal basis of the tan 1 mutant phenotype shows that this mutation disrupts the spatial regulation of cytokinesis differently than do the tonneau (ton) mutations of Arabidopsis. Examination of MT arrays in ton embryos showed that they lack PPBs altogether, which probably accounts for the abnormally shaped cells found in these embryos (Traas et al., 1995). The effects of $\tan 1$ are more reminiscent of the effects of cytochalasins, which disrupt the actin cytoskeleton and can cause cell plates to be positioned abnormally, apparently by disrupting the guidance of phragmoplasts to previously defined division sites (Palevitz and Hepler, 1974; Palevitz, 1980; Cho and Wick, 1990; Mineyuki and Palevitz, 1990; reviewed in Wick, 1991a). 
Although cytochalasins do not interfere with the formation or localization of MT PPBs in most cells examined (e.g., Eleftheriou and Palevitz, 1992), one study showed that cytochalasin treatment caused the normally asymmetric, transverse PPBs of grass guard mother cell precursors to form in more symmetric positions (M ineyuki and Palevitz, 1990). This observation suggests that actin is not essential for positioning of MT PPBs in all cells but may be important for establishing nond efault division planes as well as for phragmoplast guidance during cytokinesis.

Similarly, Tan1 is required for the establishment of longitudinal division planes and also for guidance of phragmoplasts to cortical division sites. Although our analysis did not reveal differences in actin organization between mutant and wild-type cells, the similarities between the effects of cytochalasins and the effects of the $\tan 1$ mutation lead us to speculate that the Tan 1 gene product may be involved in the mechanism by which actin influences the positioning of new cell walls. It is hoped that ongoing molecular analysis of the Tan1 gene will elucidate further the role of this gene in the spatial regulation of cytokinesis.

Finally, to explore the problem of how tanl leaves can acquire normal overall shapes despite the abnormal division behavior of their constituent cells, we also investigated how the tan 1 mutation affects arrangements of interphase cortical MTs during primordial stages of leaf development. As expected on the basis of their rectangular shapes, most interphase cells in wild-type leaf primordia have predominantly transverse cortical MTs. In mutant leaf primordia, we found a variety of cortical MT arrangements, and no rule could be devised that would allow us to predict or explain the arrangement in every cell. Nevertheless, where cells with nonaligned long axes are juxtaposed, cortical MTs tend to be coaligned across cell boundaries rather than having arrangements related to individual cell shapes.

Several previous studies have described instances in which neighboring cells have MT arrays that are coaligned (Hardham et al., 1980; Sakaguchi et al., 1988; Marc and Hackett, 1989; Hush et al., 1990; Laskowski, 1990). Such coalignment could be interpreted as suggesting that cells orient their cortical MTs (and thus their growth) partly in response to regional information of some kind that they share with their neighbors. Many factors known to influence the arrangement of interphase MTs could act on groups of cells in this way. For example, it is well known that plant hormones can rapidly alter the arrangements of interphase MTs (Shibaoka, 1991); as small molecules, hormones may diffuse so that cells in the same vicinity are exposed to similar hormone concentrations. Similarly, the application of mechanical forces and electrical fields can also reorient interphase MTs (reviewed in Williamson, 1991; Cyr, 1994; Wymer et al., 1996). Although the existence of mechanical forces and/or electrical fields within intact tissues has not been demonstrated directly, it is possible that such factors could operate on groups of neighboring cells to coordinate their MT arrangements and thus their growth patterns. Such regional influences could help orient the expansion of abnormally shaped cells during $\tan 1$ mutant leaf development so that collectively they produce organs of normal shape.

\section{METHODS}

\section{Plant Material}

A maize stock carrying the tan-pyl mutation was obtained from the Maize Genetics Cooperation Stock Center (University of Illinois, Champaign-Urbana), and the mutation subsequently was introduced into the A619 background by two successive rounds of outcrossing and selfing. Families segregating homozygous progeny derived from self-pollinated tan-py1/+ heterozygotes were grown in a greenhouse for 3 to 4 weeks to produce plant material for this study.

\section{Analysis of Cell Division Orientations}

For analysis of cell division orientations during maize leaf development, we used a whole-mount procedure to label nuclei and cell walls fluorescently with acriflavine (Sigma). Approximately $1-\mathrm{cm}$-long primordia of leaf 9 or 10 (with the outermost leaf being numbered 1) were removed from wild-type and tan-pyl segregants grown under identical conditions, sliced into $\sim 5$-mm-wide fragments, and fixed for $2 \mathrm{hr}$ to overnight at room temperature in FAA (an aqueous solution of $50 \%$ ethanol, $10 \%$ glacial acetic acid, and $3.7 \%$ formaldehyde diluted from $37 \%$ stock). After a 30 -min rinse in $15 \%$ ethanol and two 10-min rinses in distilled water $\left(\mathrm{dH}_{2} \mathrm{O}\right)$, tissues were incubated for 10 min at $60^{\circ} \mathrm{C}$ in $1 \mathrm{~N} \mathrm{HCl}$ and then rinsed for $1 \mathrm{~min}$ in $60^{\circ} \mathrm{C} \mathrm{dH}_{2} \mathrm{O}$. Tissues were then incubated in an aqueous solution containing $0.5 \%$ periodic acid for $30 \mathrm{~min}$ at room temperature and rinsed three times for $15 \mathrm{~min}$ in $\mathrm{dH}_{2} \mathrm{O}$. Tissues were then stained for at least $1 \mathrm{hr}$ in a fresh solution of acriflavine- $\mathrm{SO}_{2}(0.17 \%$ acriflavine, $0.83 \%$ potassium metabisulfite, and $0.17 \mathrm{M} \mathrm{HCl}$ in $\mathrm{dH}_{2} \mathrm{O}$ ) and washed several times in $\mathrm{dH}_{2} \mathrm{O}$ until no more color was extracted (generally overnight). Tissues were then rinsed for $5 \mathrm{~min}$ in $\mathrm{SO}_{2}-\mathrm{H}_{2} \mathrm{O}$ at room temperature (same as acriflavine- $\mathrm{SO}_{2}$ but without acriflavine) and then cleared in chloral hydrate $\left(2.5 \mathrm{mg} / \mathrm{mL}\right.$ in $\left.\mathrm{dH}_{2} \mathrm{O}\right)$ for at least $2 \mathrm{hr}$ or until a suitable staining intensity was obtained. Tissues were then mounted in chloral hydrate, and fluorescence of epidermal nuclei and cell walls was viewed on a laser scanning confocal microscope system (Carl Zeiss, Inc., Thornwood, NY) with a $40 \times$ oil immersion objective. Acriflavine was excited with the 488-nm line of an ArKr laser, and images were collected using a standard fluorescein isothiocyanate (FITC) filter set and emission filter LP 515.

Midplane optical sections from at least 10 fields of view were collected from dispersed areas for each of three wild-type and three mutant individuals. To analyze cell division orientations, we examined images to identify all walls that were stained less intensely than the walls they adjoined at both ends, indicating a lower content of cellulose. More than 1000 recently formed cell walls were analyzed in each data set (wild type and mutant). A line was drawn joining the two end points of each new wall, and a second line was drawn corresponding to the long axis of the mother cell or its best approximation. For each cell wall analyzed, the angle between these two lines was measured to obtain the data plotted in Figure $2 \mathrm{C}$. The FisherBehrens test for comparison of two populations with unequal variances (Campbell, 1989) was used to analyze the statistical significance of the 
differences between the angles in wild type and mutant primordia that are shown in Figure $2 \mathrm{C}$.

\section{Labeling and Analysis of Microtubules and Nuclei (DNA)}

One leaf primordium was removed from each homozygous tan-pyl mutant or wild-type sibling grown at the same time under the same conditions. The leaf primordium selected was either leaf 9 or 10 and was 0.6 to $1.6 \mathrm{~cm}$ in length. Each leaf was cut into longitudinal segments of $\sim 1 \mathrm{~mm}$ in width and up to $1 \mathrm{~cm}$ in length and placed into a small vial containing fixative solution. Leaf segments were fixed for 3 to $4 \mathrm{hr}$ with gentle shaking in $4 \%$ paraformaldehyde (Polysciences, Warrington, PA), $0.1 \%$ Triton X-100, 2\% glycerol, and 5\% DMSO in PHEM buffer (60 mM Pipes, $25 \mathrm{mM}$ Hepes, $10 \mathrm{mM}$ EGTA, and $4 \mathrm{mM}$ $\mathrm{MgCl}_{2}, \mathrm{pH}$ 6.9). All steps were performed at room temperaure. Segments were rinsed for $15 \mathrm{~min}$ in PHEM buffer with 5\% DMSO (PHEM-DMSO) and digested for $15 \mathrm{~min}$ in an aqueous solution of $1 \%$ pectolyase (Seishin Pharmaceuticals, Tokyo, Japan) and 2\% driselase (Sigma). After a 10-min rinse in PHEM-DMSO, segments were extracted for $1 \mathrm{hr}$ in 1\% Triton-X100 in PHEM-DMSO, rinsed for $5 \mathrm{~min}$ in PHEM, and then rinsed for $10 \mathrm{~min}$ in PBS. Segments were then incubated overnight with gentle shaking in anti- $\beta$-tubulin antibody (Amersham, Arlington Heights, IL) diluted 1:600 in PBS with 1 $\mathrm{mg} / \mathrm{mL}$ BSA (Sigma) (PBS-BSA). Segments were rinsed for up to $1 \mathrm{hr}$ in PBS, then incubated for 6 to $7 \mathrm{hr}$ in FITC-conjugated anti-mouse lg (Sigma) diluted 1:100 in PBS-BSA, and rinsed in PBS (10 min); the nuclei were counterstained with an aqueous solution of $5 \mu \mathrm{g} / \mathrm{mL}$ propidium iodide (PI; Sigma) for $30 \mathrm{~min}$. After a final rinse in PBS, leaf segments were mounted in $10 \%$ Mowiol (Calbiochem, La J olla, CA) containing $1 \%$ p-phenylenediamine (Sigma). These preparations could be stored for at least 1 week at $4^{\circ} \mathrm{C}$ in the dark without noticeable deterioration.

Images of microtubules and DNA were collected on the Zeiss laser scanning confocal system by using a $63 \times$ oil immersion objective. Fluorochromes were excited successively using either the 488-nm (FITC) or 568-nm (PI) lines from an ArKr laser. Each emission wavelength passed through a double dichroic mirror (FT 488/568) and was selected for imaging using the dichroic mirror DCLP 560. FITC images were collected using emission filter BP 515-540, and PI images were collected using emission filter LP590. Surface and midplane optical sections for each fluorochrome were collected for at least 15 fields of view representing dispersed areas of four mutant and four wild-type leaf primordia. This collection of images was used to reconstruct in each field of view the shape, nuclear position, and microtubule (MT) array present in every cell containing a preprophase band (PPB), spindle, or phragmoplast.

More than 1000 such dividing cells from each data set (wild type and mutant) were analyzed. For each dividing cell, we drew a reference line down the middle to represent the long axis or its best approximation. A second line was drawn to represent the orientation of the MT array; this line was parallel to PPBs and phragmoplasts and perpendicular to the spindle axes. For phragmoplasts that were curved or were elongated such that only their edges contained MTs, a straight line was drawn through the two ends of the phragmoplast to assign its orientation. For each MT array, the angle between the two lines was determined to obtain the angles plotted in Figure 4. A distinction was made between early phragmoplasts $(>2 \mu \mathrm{m}$ from the parent cell wall at one or both ends) and late phragmoplasts $(<2 \mu \mathrm{m}$ from the parental wall at both ends). Because late phragmoplasts were considered to be a better indicator of the final position of the cell plate, data for this category only were included in Figure 4C. The Fisher-Behrens test for comparison of two populations with unequal variances (Campbell, 1989) was used to analyze the statistical significance of results for wild-type and mutant cells.

\section{Double Labeling of F-Actin and MTs}

Although labeling of tissue segments for actin with FITC-phalloidin alone, as described by Cleary and Mathesius (1996), produced excellent images of cortical and cytoplasmic actin, interpretation of these images required additional information to determine cell cycle stage and nuclear position. Counterstaining of FITC-phalloidinlabeled tissues with PI was not effective in most cells, because PI staining was restricted to the periphery of tissue segments and quenched the actin labeling. Therefore, to analyze the formation and position of actin arrays, we developed a procedure for double labeling F-actin and MTs. For this analysis, primordia of wild-type and mutant leaves 10 to 14 (0.8 to $1.4 \mathrm{~cm}$ in length) were used. Tissue preparation for staining was as described in Labeling and Analysis of Microtubules and Nuclei (DNA), except that fixation was for 1 to $2 \mathrm{hr}$ and the buffer used was PM5E (50 mM Pipes, $5 \mathrm{mM}$ EGTA, and 2 $\mathrm{mM} \mathrm{MgSO}{ }_{4}$ ). The addition of $5 \%$ DMSO to the fixative solution was necessary for preservation of MTs and had no detrimental effects on actin. Treatment of the segments with $200 \mu \mathrm{M}$ 3-maleimidobenzoyl $\mathrm{N}$-hydroxysuccinimide ester before fixation had no beneficial effects on actin preservation and thus was not used.

The fixed, digested, detergent-extracted leaf segments were incubated overnight at room temperature in a mixture of B512 anti$\alpha$-tubulin antibody (Sigma) and Texas Red-conjugated phalloidin (Molecular Probes, Inc., Eugene, OR). To prepare this staining solution, we evaporated methanol from the appropriate volume of a 6.6 $\mu \mathrm{M}$ Texas Red-phalloidin methanol stock solution. The precipitated Texas Red-phalloidin was then redissolved to a final concentration of $0.66 \mu \mathrm{M}$ in anti- $\alpha$-tubulin antibody diluted 1:1500 in PBS-BSA with $0.02 \% \mathrm{NaN}_{3}$. After staining, leaf segments were rinsed in PBS (15 $\mathrm{min}$ ) and incubated for 5 to $7 \mathrm{hr}$ in a mixture of $0.66 \mu \mathrm{M}$ Texas Redphalloidin with FITC-conjugated sheep anti-mouse Ig antibody (Silenus, Hawthorn, Australia) diluted 1:30 in PBS-BSA with $0.02 \% \mathrm{NaN}_{3}$ prepared as described above. Segments were rinsed in PBS (15 min) and mounted as described above.

Double labeling of MTs and actin was observed using a $63 \times \mathrm{NA}$ oil immersion objective on a Bio-Rad MRC-600 confocal laser scanning system fitted with an ArKr laser and coupled to a Zeiss Axiovert microscope. Surface and midplane optical sections of FITC-labeled MTs (excitation at $488 \mathrm{~nm}$; BHS filter block) and Texas Red-labeled actin (excitation at $568 \mathrm{~nm}$; YHS filter block) were recorded successively for each field of view (filter blocks; BioRad). In general, the best MT labeling was found near the edges of the tissue segments, whereas the best actin labeling was found toward the center. However, sufficient overlap between MT and actin staining was obtained to allow analysis of both images in $>100$ cells (wild type and mutant combined) containing a PPB, spindle, or phragmoplast.

\section{ACKNOWLEDGMENTS}

We gratefully acknowledge financial support for this work from the National Institutes of Health (Grant No. R01-GM 53137 to L.G.S.) and 
from a U.S. Department of Agriculture/National Science Foundation/ Department of Energy Interagency grant to the Cytonet Group, which supported our collaboration. We thank the Maize Genetics Cooperation Stock Center for seeds; Kim Gallagher, J ocelyn Malamy, and Erik Vollbrecht for advice and help with the acriflavine staining procedure; Susan Whitfield for help with preparing figures; Tony Perdue for help with confocal microscopy; Margaret Sammut for technical assistance with the MT/actin labeling; and Dawn Franklin for help with the data analysis. We are grateful for stimulating and helpful discussions with Adrienne Hardham, Brian Gunning, Paul Green, and Richard Cyr, and thank J ohn Fowler, Brian Gunning, and Kim Gallagher for helpful comments on the manuscript.

Received March 30, 1998; accepted September 8, 1998.

\section{REFERENCES}

Baluska, F., Vitha, S., Barlow, P.W., and Volkmann, D. (1997). Rearrangements of $\mathrm{F}$-actin arrays in growing cells in intact maize root apex tissues: A major developmental switch occurs in the postmitotic transition region. Eur. J. Cell Biol. 72, 113-121.

Campbell, R.C. (1989). Statistics for Biologists, 3rd ed. (Cambridge, UK: Cambridge University Press).

Cho, S.-O., and Wick, S.M. (1989). Microtubule orientation during stomatal differentiation in grasses. J. Cell Sci. 92, 581-594.

Cho, S.-O., and Wick, S.M. (1990). Distribution and function of actin in the developing stomatal complex of winter rye (Secale cereale cv. Puma). Protoplasma 157, 154-164.

Cleary, A.L. (1995). F-actin redistributions at the division site in living Tradescantia stomatal complexes as revealed by microinjection of rhodamine-phalloidin. Protoplasma 185, 152-165.

Cleary, A.L., and Mathesius, U. (1996). Rearrangements of F-actin during stomatogenesis visualised by confocal microscopy in fixed and permeabilised Tradescantia leaf epidermis. Bot. Acta 109, 15-24.

Cleary, A.L., Gunning, B.E.S., Wasteneys, G.O., and Hepler, P.K. (1992). Microtubule and F-actin dynamics at the division site in living Tradescantia stamen hair cells. J. Cell Sci. 103, 977-988.

Cyr, R.J . (1994). Microtubules in plant morphogenesis: Role of the cortical array. Annu. Rev. Cell Biol. 10, 153-180.

Eleftheriou, E.P., and Palevitz, B.A. (1992). The effect of cytochala$\sin D$ on preprophase band organization in root tip cells of Allium. J. Cell Sci. 103, 989-998.

Flanders, D.J ., Rawlins, D.J ., Shaw, P.J ., and Lloyd, C.W. (1990). Nucleus-associated microtubules help determine the division plane of plant epidermal cells: Avoidance of four-way junctions and the role of cell geometry. J . Cell Biol. 110, 1111-1122.

Gunning, B.E.S. (1982). The cytokinetic apparatus: Its development and spatial regulation. In The Cytoskeleton in Plant Growth and Development, C.W. Lloyd, ed (London: Academic Press), pp. 229-292.

Gunning, B.E.S., and Wick, S.M. (1985). Preprophase bands, phragmoplasts, and spatial control of cytokinesis. J. Cell Sci. 2 (suppl.), 157-179.
Hardham, A.R., Green, P.B., and Lang, J.M. (1980). Reorganization of cortical microtubules and cellulose deposition during leaf formation in Graptopetalum paraguayense. Planta 149, 181-195.

Hush, J.M., Hawes, C.R., and Overall, R.L. (1990). Interphase microtubule re-orientation predicts a new cell polarity in wounded pea roots. J . Cell Sci. 96, 47-61.

Kakimoto, T., and Shibaoka, H. (1987). Actin filaments and microtubules in the preprophase band and phragmoplast of tobacco cells. Protoplasma 140, 151-156.

Laskowski, M.J . (1990). Microtubule orientation in pea stem cells: A change in orientation follows the initiation of growth rate decline. Planta 181, 44-52.

Liu, B., and Palevitz, B.A. (1992). Organization of cortical microfilaments in dividing root cells. Cell Motil. Cytoskeleton 23, 252-264.

Lloyd, C.W. (1991). Cytoskeletal elements of the phragmosome establish the division plane in vacuolated higher plant cells. In The Cytoskeletal Basis of Plant Growth and Form, C.W. Lloyd, ed (London: Academic Press), pp. 245-257.

Marc, J ., and Hackett, W.P. (1989). A new method for immunofluorescent localization of microtubules in surface cell layers: Application to the shoot apical meristem of Hedera. Protoplasma 148, 70-79.

McCurdy, D.W., and Gunning, B.E.S. (1990). Reorganization of cortical actin microfilaments and microtubules at preprophase and mitosis in wheat root-tip cells: A double label immunofluorescence study. Cell Motil. Cytoskeleton 15, 76-87.

Mineyuki, Y., and Gunning, B.E.S. (1990). A role for preprophase bands of microtubules in maturation of new cell walls, and a general proposal on the function of preprophase band sites in cell division in higher plants. J . Cell Sci. 97, 527-537.

Mineyuki, Y., and Palevitz, B.A. (1990). Relationship between preprophase band organization, $\mathrm{F}$-actin and the division site in Allium. J . Cell Sci. 97, 283-295.

Ota, T. (1961). The role of cytoplasm in cytokinesis of plant cells. Cytologia 26, 428-447.

Palevitz, B.A. (1980). Comparative effects of phalloidin and cytochalasin B on motility and morphogenesis in Allium. Can. J. Bot. 58, 773-785.

Palevitz, B.A. (1986). Division plane determination in guard mother cells of Allium: Video time-lapse analysis of nuclear movements and phragmoplast rotation in the cortex. Dev. Biol. 117, 644-654.

Palevitz, B.A. (1987). Actin in the preprophase band of Allium cepa. J. Cell Biol. 104, 1515-1519.

Palevitz, B.A., and Hepler, P.K. (1974). The control of the plane of division during stomatal differentiation in Allium. II. Drug studies. Chromosoma 46, 327-341.

Pickett-Heaps, J.E., and Northcote, D.H. (1966). Cell division in the formation of the stomatal complex of the young leaves of wheat. J . Cell Sci. 1, 121-128.

Sakaguchi, S., Hogetsu, T., and Hara, N. (1988). Arrangement of cortical microtubules in the shoot apex on Vinca major L. Planta 175, 403-411.

Shevell, D.E., Leu, W.-M., Gillmor, C.S., Xia, G., Feldmann, K.A., and Chua, N.-H. (1994). EMB30 is essential for normal cell division, cell expansion and cell adhesion in Arabidopsis and encodes a protein that has similarity to Sec7. Cell 77, 1051-1062. 
Shibaoka, H. (1991). Microtubules and the regulation of cell morphogenesis by plant hormones. In The Cytoskeletal Basis of Plant Growth and Form, C.W. Lloyd, ed (London: Academic Press), pp. 159-168.

Smith, L.G., Hake, S., and Sylvester, A.W. (1996). The tangled1 mutation alters cell division orientations throughout maize leaf development without altering leaf shape. Development 122, 481-489.

Staehelin, L.A., and Hepler, P.K. (1996). Cytokinesis in higher plants. Cell 84, 821-824.

Suttle, A.D. (1924). Genetic Interrelations of Different Types of Dwarf Corn. PhD Dissertation (Ithaca, NY: Cornell University).

Sylvester, A.W., Cande, W.Z., and Freeling, M. (1990). Division and differentiation during normal and liguleless1 maize leaf development. Development 110, 985-1000.

Traas, J .A., Doonan, J .H., Rawlins, D.J ., Shaw, P., Watts, J ., and Lloyd, C.W. (1987). An actin network is present in the cytoplasm throughout the cell cycle of carrot cells and associates with the dividing nucleus. J. Cell Biol. 105, 387-395.

Traas, J .A., Bellini, C., Nacry, P., Kronenberger, J ., Bouchez, D., and Caboche, M. (1995). Normal differentiation patterns in plants lacking microtubular preprophase bands. Nature 375, 676-677.

Wick, S.M. (1991a). Spatial aspects of cytokinesis in plant cells. Curr. Opin. Cell Biol. 3, 253-260.

Wick, S.M. (1991b). The preprophase band. In The Cytoskeletal Basis of Plant Growth and Form, C.W. Lloyd, ed (London: Academic Press), pp. 231-244.

Williamson, R.E. (1991). Orientation of cortical microtubules in interphase plant cells. Int. Rev. Cytol. 129, 135-206.

Wymer, C.L., Wymer, S.A., Cosgrove, D.J ., and Cyr, R.J . (1996). Plant cell growth responds to external forces and the response requires intact microtubules. Plant Physiol. 110, 425-430. 
The Tangled1 Gene Is Required for Spatial Control of Cytoskeletal Arrays Associated with Cell Division during Maize Leaf Development

Ann L. Cleary and Laurie G. Smith

Plant Cell 1998;10;1875-1888

DOI 10.1105/tpc.10.11.1875

This information is current as of July 22, 2020

References

Permissions

eTOCs

CiteTrack Alerts

Subscription Information
This article cites 35 articles, 13 of which can be accessed free at: /content/10/11/1875.full.html\#ref-list-1

https://www.copyright.com/ccc/openurl.do?sid=pd_hw1532298X\&issn=1532298X\&WT.mc_id=pd_hw1532298X

Sign up for eTOCs at:

http://www.plantcell.org/cgi/alerts/ctmain

Sign up for CiteTrack Alerts at:

http://www.plantcell.org/cgi/alerts/ctmain

Subscription Information for The Plant Cell and Plant Physiology is available at:

http://www.aspb.org/publications/subscriptions.cfm 